\title{
Orbital mixing at the onset of high-temperature superconductivity in $\mathrm{FeSe}_{1-x} \mathrm{Te}_{x} / \mathrm{CaF}_{2}$
}

\author{
K. Nakayama $\odot,{ }^{1,2}$ R. Tsubono, ${ }^{1}$ G. N. Phan,,${ }^{1, *}$ F. Nabeshima, ${ }^{3}$ N. Shikama $\odot,{ }^{3}$ T. Ishikawa, ${ }^{3}$ Y. Sakishita, ${ }^{3}$ S. Ideta,,${ }^{4,5}$ \\ K. Tanaka $\odot,^{4,5}$ A. Maeda, ${ }^{3}$ T. Takahashi, ${ }^{1,6,7}$ and T. Sato ${ }^{1,6,7}$ \\ ${ }^{1}$ Department of Physics, Tohoku University, Sendai 980-8578, Japan \\ ${ }^{2}$ Precursory Research for Embryonic Science and Technology, Japan Science and Technology Agency, Tokyo 102-0076, Japan \\ ${ }^{3}$ Department of Basic Science, University of Tokyo, 3-8-1 Komaba, Meguro, Tokyo 153-8902, Japan \\ ${ }^{4}$ UVSOR Synchrotron Facility, Institute for Molecular Science, Okazaki 444-8585, Japan \\ ${ }^{5}$ School of Physical Sciences, The Graduate University for Advanced Studies (SOKENDAI), Okazaki 444-8585, Japan \\ ${ }^{6}$ Center for Spintronics Research Network, Tohoku University, Sendai 980-8577, Japan \\ ${ }^{7}$ WPI Research Center, Advanced Institute for Materials Research, Tohoku University, Sendai 980-8577, Japan
}

(Received 15 September 2020; revised 5 January 2021; accepted 7 January 2021; published 20 January 2021)

\begin{abstract}
We perform systematic high-resolution angle-resolved photoemission spectroscopy of iron-chalcogenide superconductor $\mathrm{FeSe}_{1-x} \mathrm{Te}_{x}$ films on $\mathrm{CaF}_{2}$ which exhibit a unique paramagnetic nematicity at $x=0$ (pristine $\mathrm{FeSe})$ and a gigantic $T_{\mathrm{c}}$ enhancement at the critical Te concentration $\left(x_{\mathrm{c}}\right)$ of $x \sim 0.2$. Upon increasing the Te concentration from $x=0$, the electronlike Fermi-surface shape at the Brillouin-zone corner shows a clear change associated with a remarkable energy shift of the $d_{x z / y z}$ orbital, indicative of the suppression of nematicity near $x_{\mathrm{c}}$. Evolution of band structure at the Brillouin-zone center is characterized by a drastic upward shift of the $d_{x y}$ band with increasing $x$, leading to an orbital switching from $d_{x z / y z}$ to $d_{x z / y z}+d_{x y}$ accompanied by a mass enhancement. These results demonstrate that the pristine and high- $T_{\mathrm{c}} \mathrm{FeSe}_{1-x} \mathrm{Te}_{x}$ have distinctly different electronic structures. The present study lays the foundation for understanding the origin of high- $T_{\mathrm{c}}$ superconductivity and the interplay with electronic nematicity.
\end{abstract}

DOI: 10.1103/PhysRevResearch.3.L012007

Iron-based high-temperature $T_{\mathrm{c}}$ superconductors are a multiorbital system, in which all five $\mathrm{Fe} 3 d$ orbitals are involved in the low-energy electronic structure. As an emergent phenomenon associated with the multiorbital nature, the electronic nematicity, defined as a spontaneous breaking of rotational symmetry [1-8], is at the forefront of research in condensed-matter physics. The nematic state is widely observed near the superconducting state in iron-based superconductors, so particular interest is focused on the interplay between nematicity and superconductivity. However, the nematicity is strongly coupled to the collinear-type antiferromagnetic order accompanied by a tetragonal-to-orthorhombic structural transition [9], which makes it difficult to isolate the influence of nematicity from antiferromagnetism.

Among iron-based superconductors, FeSe [10,11] is a unique system showing nematicity without antiferromagnetic order. Because of this unique property, recent studies on FeSe have revealed several important insights into nematicity and the impact on superconductivity, such as a large energy shift

*Present address: Beijing National Laboratory for Condensed Matter Physics and Institute of Physics, Chinese Academy of Sciences, Beijing 100080, China.

Published by the American Physical Society under the terms of the Creative Commons Attribution 4.0 International license. Further distribution of this work must maintain attribution to the author(s) and the published article's title, journal citation, and DOI. of the $d_{x z}$ and $d_{y z}$ bands due to the lifting of orbital degeneracy around the structural transition temperature $T_{\mathrm{s}} \sim 90 \mathrm{~K}$ [12-17] and a signature of orbital-selective pairing below $T_{\text {c }}$ [18-21]. FeSe also has a great advantage in tunability of superconductivity through chemical substitution [22-24], high-pressure application [25,26], and/or carrier doping [27-29]. Therefore, one can investigate the interplay between nematicity and superconductivity by precisely tracing the evolution of the electronic structure responsible for nematicity and superconductivity while controlling some physical parameters. In this respect, isovalent-substituted $\mathrm{FeSe}_{1-x} \mathrm{Te}_{x}$ films grown on $\mathrm{CaF}_{2}$ substrate $\left(\mathrm{FeSe}_{1-x} \mathrm{Te}_{x} / \mathrm{CaF}_{2}\right)$ offer an excellent platform because the nematicity without antiferromagnetic order is realized in $\mathrm{FeSe} / \mathrm{CaF}_{2}(x=0)$ as in bulk FeSe [30,31] and the highest $T_{\mathrm{c}}$ of $23 \mathrm{~K}$ among non-carrierdoped FeSe-based compounds has been reported at the critical Te concentration $x_{\mathrm{c}}$ of $\sim 0.2$ at ambient pressure $[23,24,32]$ [Fig. 1(a)] (note that bulk $\mathrm{FeSe}_{1-x} \mathrm{Te}_{x}$ crystals of $0.1<x<$ 0.4 were unavailable due to the phase separation [22]). It is thus desirable to determine experimentally the electronic structure of $\mathrm{FeSe}_{1-x} \mathrm{Te}_{x} / \mathrm{CaF}_{2}$ as a function of $x$.

Angle-resolved photoemission spectroscopy (ARPES) is the best suited technique to probe the momentum-resolved electronic structure. However, there is no systematic ARPES report on $\mathrm{FeSe}_{1-x} \mathrm{Te}_{x} / \mathrm{CaF}_{2}$ with a series of different $x$ values, mainly due to the difficulties in fabricating $\mathrm{FeSe}_{1-x} \mathrm{Te}_{x} / \mathrm{CaF}_{2}$ and in preparing a clean surface necessary for ARPES measurements. In this Letter, by overcoming these difficulties, we report a systematic ARPES study on the evolution of 
(a) $\quad \mathrm{FeSe}_{1-x} \mathrm{Te}_{x} / \mathrm{CaF}_{2}$

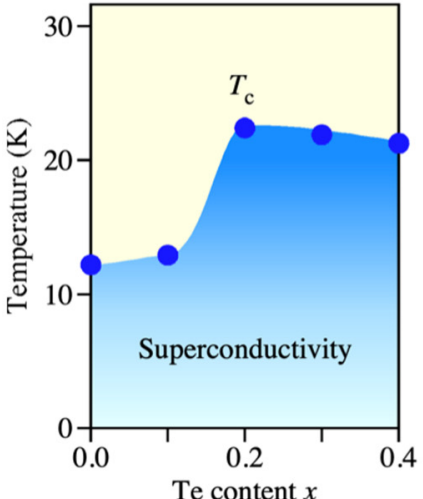

(b)

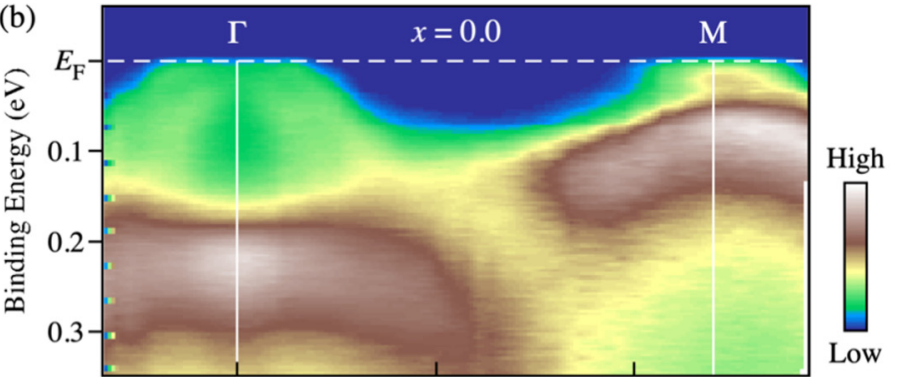

(c)

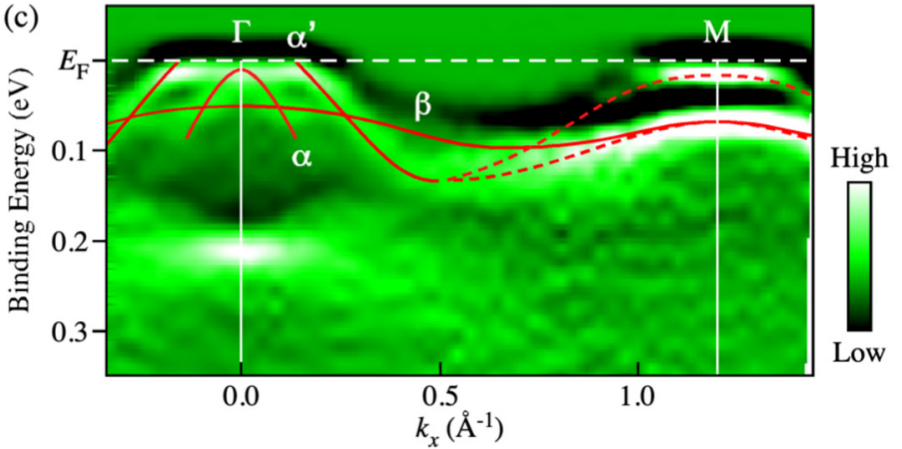

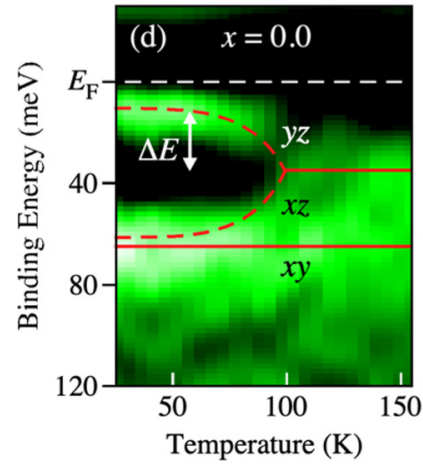
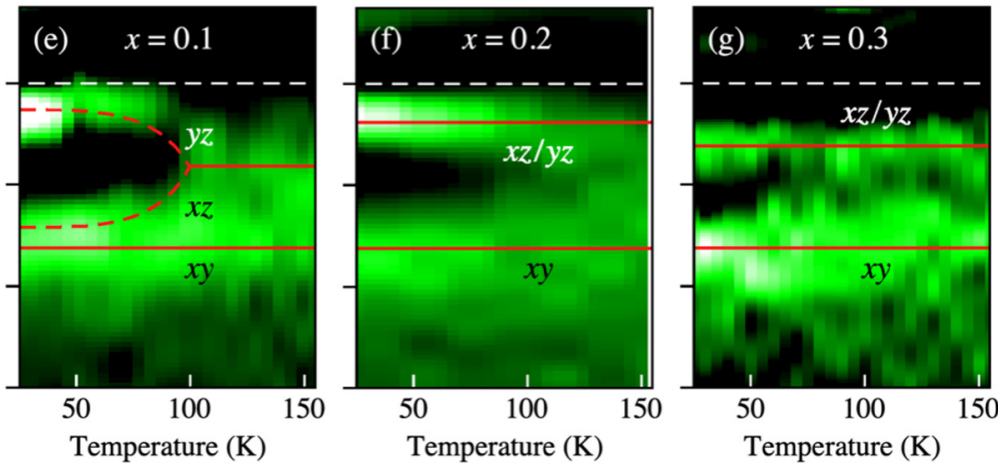

FIG. 1. (a) Phase diagram of $\mathrm{FeSe}_{1-x} \mathrm{Te}_{x} / \mathrm{CaF}_{2}$ as a function of Te concentration $x$ [23]. Also shown are plots of (b) the APRES intensity and (c) the corresponding second-derivative intensity, measured along the $\Gamma-M$ line at $T=30 \mathrm{~K}$ for FeSe $/ \mathrm{CaF}_{2}(x=0)$ with linearly polarized 21.2-eV photons. The temperature dependence of the second-derivative ARPES intensity at the $M$ point is shown for (d) $x=0$, (e) $x=0.1$, (f) $x=0.2$, and (g) $x=0.3$. The red curves in (c) $-(\mathrm{g})$ are a guide for the eyes to trace the band dispersions.

the electronic structure in $\mathrm{FeSe}_{1-x} \mathrm{Te}_{x} / \mathrm{CaF}_{2}$ over a wide $x$ range $(0 \leqslant x \leqslant 0.4)$. We reveal the suppression of nematicity on approaching $x_{\mathrm{c}}$, indicative of the anticorrelation between nematicity and superconductivity. We also find evidence for the electronic reconstruction near the Fermi level $E_{\mathrm{F}}$ characterized by switching of the $\mathrm{Fe} 3 d$ orbital character. We discuss implications of our observation in relation to the origin of $T_{\mathrm{c}}$ enhancement and the suppression of nematicity.

High-quality $\mathrm{FeSe}_{1-x} \mathrm{Te}_{x}$ films $(x=0-0.4)$ with a thickness of approximately 400 unit cells were grown on $\mathrm{CaF}_{2}$ by pulsed-laser-deposition method. These films are compressively strained, so $\mathrm{FeSe} / \mathrm{CaF}_{2}(x=0)$ shows a higher $T_{\mathrm{c}}$ $(12 \mathrm{~K})$ than the bulk counterpart $(\sim 9 \mathrm{~K})$ [33]. Details of film growth and their characterization are described elsewhere [23,32-34]. The ARPES measurements were performed with MBS-A1 and Scienta-Omicron-SES2002 electron analyzers at BL7U in UVSOR and Tohoku University, respectively. We used 21.2-eV photons. The energy and angular resolutions were set to be $12-30 \mathrm{meV}$ and $0.2^{\circ}-0.3^{\circ}$, respectively. A clean surface for ARPES measurements was obtained by cleaving the films in situ in an ultrahigh vacuum. We note that
$\mathrm{FeSe}_{1-x} \mathrm{Te}_{x} / \mathrm{CaF}_{2}$ is often cleaved at the interface between $\mathrm{FeSe}_{1-x} \mathrm{Te}_{x}$ and $\mathrm{CaF}_{2}$ and the resultant exposure of the $\mathrm{CaF}_{2}$ surface prevents us from obtaining the ARPES data for the $\mathrm{FeSe}_{1-x} \mathrm{Te}_{x}$ layer. Nevertheless, we have cleaved more than 70 samples and obtained a clean $\mathrm{FeSe}_{1-x} \mathrm{Te}_{x}$ surface.

Figures 1(b) and 1(c) show plots of the ARPES intensity and corresponding second-derivative intensity, respectively, along the $\Gamma-M$ line of the Brillouin zone at $T=30 \mathrm{~K}$ for pristine $\mathrm{FeSe} / \mathrm{CaF}_{2}\left(x=0\right.$ and $\left.T_{\mathrm{c}}=12 \mathrm{~K}\right)$. The near- $E_{\mathrm{F}}$ band structure around the $\Gamma$ point consists of two highly dispersive bands $\alpha$ and $\alpha^{\prime}$ and a relatively flat band $\beta$ [see red curves in Fig. 1(c)]. According to previous studies, the $\alpha$ and $\alpha^{\prime}$ bands are ascribed to the $\mathrm{Fe} 3 d_{x z} / d_{y z}$ orbitals and the $\beta$ band is ascribed to the Fe $3 d_{x y}$ orbital [12-17,30]. Around the $M$ point, there are two holelike bands topped at binding energies $E_{\mathrm{B}}$ of $\sim 20$ and $\sim 70 \mathrm{meV}$, which are attributed to the $d_{y z}$ - and $d_{x z}$-derived bands originating from a splitting of the $\alpha^{\prime}$ band due to the lifting of orbital degeneracy [see red dashed curves in Fig. 1(c)] [12-17] because $\mathrm{FeSe} / \mathrm{CaF}_{2}$ is in the nematic state at $30 \mathrm{~K}[30,31]$. In fact, a temperaturedependent study at the $M$ point reveals a large downward shift 

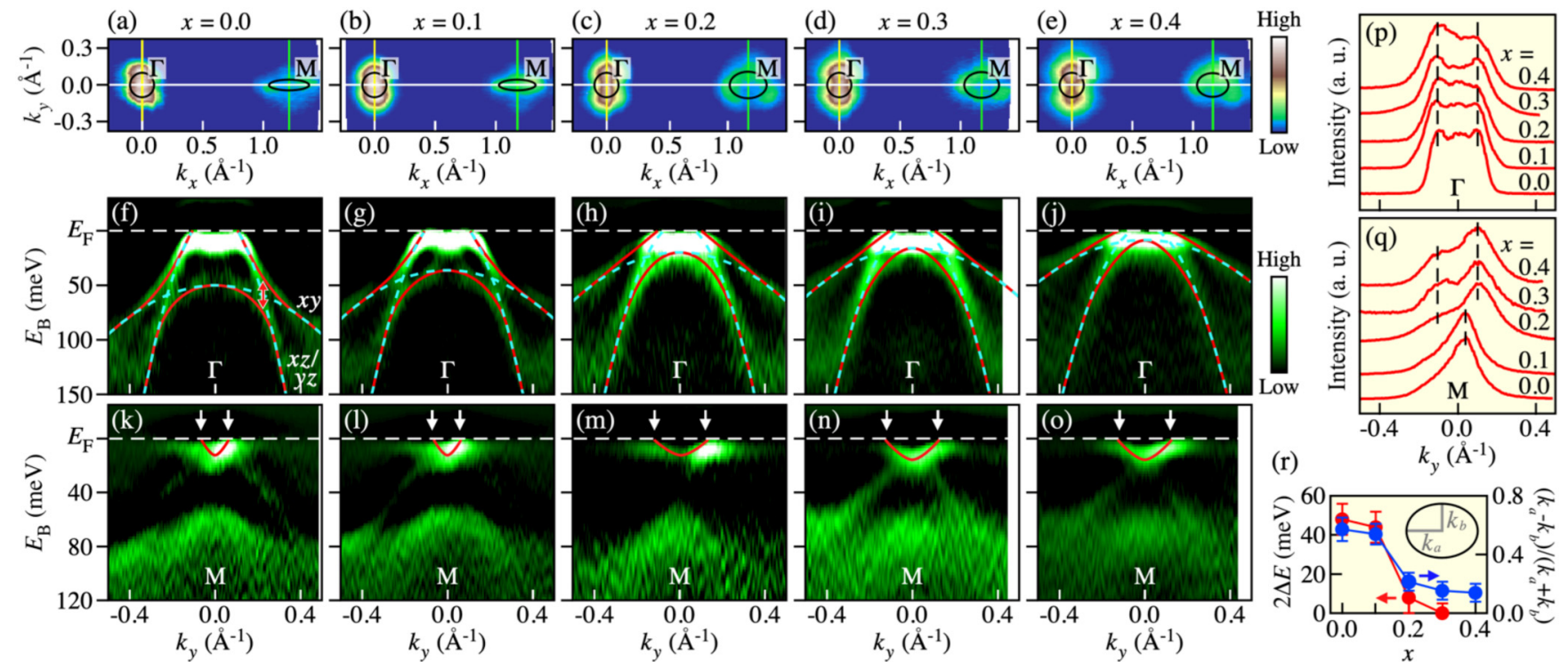

FIG. 2. Te-concentration dependence of the ARPES-intensity map at $E_{\mathrm{F}}$ as a function of the two-dimensional wave vector for $\mathrm{FeSe}_{1-x} \mathrm{Te}_{x} / \mathrm{CaF}_{2}$, with (a) $x=0.0$, (b) $x=0.1$, (c) $x=0.2$, (d) $x=0.3$, and (e) $x=0.4$, at $T=30 \mathrm{~K}$. Black curves are the schematic of FSs. Also shown is the near- $E_{\mathrm{F}}$ second-derivative ARPES intensity obtained at $T=30 \mathrm{~K}$ along yellow and green lines, around the (f)-(j) $\Gamma$ and (k)-(o) $M$ points, in (a)-(e), respectively. Cyan and red curves are a guide for the eyes to trace the band dispersions before and after hybridization, respectively. The band dispersions before the hybridization were estimated by extrapolating the dispersions determined in the $(E, \mathbf{k})$ region away from the intersection point. (p) and (q) Comparison of momentum distribution curves at $E_{\mathrm{F}}$ extracted from (f)-(j) and (k)-(o), respectively. Dashed lines are a guide for the eyes to trace the peak position. (r) Te-concentration dependence of an anisotropy of the electronlike FS $\left(k_{a}-k_{b}\right) /\left(k_{a}+k_{b}\right)$ (blue circles), compared with the nematic energy scale $2 \Delta E$ (red circles) approximated as twice the energy difference of the $d_{y z}$ band between $T=30$ and $110 \mathrm{~K}$.

( $\Delta E=24 \mathrm{meV}$ ) of the $d_{y z}$ band with increasing temperature [Fig. 1(d)], consistent with the occurrence of nematicity (note that an upward shift expected for the $d_{x z}$ counterpart is not clearly resolved due to an energy overlap with the $\beta$ band at higher $E_{\mathrm{B}}$ ). To clarify the evolution of nematicity with Te substitution, we performed temperature-dependent studies at the $M$ point for $x=0.1-0.3$. A side-by-side comparison [Figs. $1(\mathrm{~d})-1(\mathrm{~g})]$ reveals that the $d_{y z}$ band shows a large energy shift with increasing temperature at $x=0.1$, similarly to the observation at $x=0$, whereas the $d_{y z}$ band is almost stationary with temperature at $x=0.2$ and 0.3 . This strongly suggests that the nematic state is destroyed at $x \sim x_{\mathrm{c}}$. The suppression of nematicity while approaching $x=x_{\mathrm{c}}$ demonstrates the anticorrelation between nematicity and $T_{\mathrm{c}}$ in $\mathrm{FeSe}_{1-x} \mathrm{Te}_{x} / \mathrm{CaF}_{2}$.

To investigate how the band structure changes across $x_{\mathrm{c}}$, we performed systematic measurements of the Fermi surface (FS) [Figs. 2(a)-2(e)] and the band dispersion [Figs. 2(f)2(o)] for five different compositions. One can recognize that the FS shape around the $M$ point is elongated along the $k_{x}$ axis for $x \leqslant 0.1$, whereas it is more rounded for $x \geqslant 0.2$ [Figs. 2(a)-2(e)]. This change is fairly drastic, as evidenced by a jump in the Fermi wave vector $k_{\mathrm{F}}$ of the electron-band dispersion between $x=0.1$ and 0.2 [see white arrows in Figs. $2(\mathrm{k})-2(\mathrm{o})$, the peak position of the momentum distribution curves at $E_{\mathrm{F}}$ in Fig. 2(q), and Fig. S1 in the Supplemental Material [35]]. To evaluate the FS anisotropy quantitatively, we assumed an elliptical shape for the electronlike FS and defined $k_{a}$ and $k_{b}$ as the $k_{\mathrm{F}}$ value along the major and minor axes, respectively [see the inset to Fig. 2(r)], to calculate a value of $\left(k_{a}-k_{b}\right) /\left(k_{a}+k_{b}\right)$ that gives a large (small) value when the FS is elongated (circular). The strong variation obtained at $x \sim 0.2$ [see red circles in Fig. 2(r)] confirms that the electronlike FS has a different shape between $x<x_{\mathrm{c}}$ and $x \geqslant x_{\mathrm{c}}$. Further, the $x$ dependence of the FS anisotropy follows that of the estimated nematic energy scale $2 \Delta E$, which corresponds to twice the $d_{y z}$-band energy shift in Figs. $1(\mathrm{~d})-1(\mathrm{~g})$ [blue circles in Fig. 2(r)], indicating that the FS anisotropy is linked to the presence/absence of nematicity, namely, the elongated shape of FS is triggered by the upward shift of the $d_{y z}$ orbital [12-17].

To clarify the effect of Te substitution in more detail, we turn our attention to the Brillouin-zone center. It has been reported that, although the $d_{x z / y z}$-orbital degeneracy is lifted around the $\Gamma$ point by nematicity as in the case of the $M$ point, the energy difference between the $d_{x z}$ and $d_{y z}$ bands and the resultant change in the FS shape are relatively small $[14,16,17]$. Therefore, there is no discernible change in the holelike-FS shape within the present experimental accuracy [Figs. 2(a)-2(e) and 2(p)]. Instead, a remarkable $x$ dependence beyond the effect of nematicity manifests itself in the band dispersion [Figs. 2(f)-2(j)]. At $x=0$, there are a highly dispersive $d_{x z / y z}$-band crossing $E_{\mathrm{F}}$ and a relatively flat $d_{x y}$ band with the top of the dispersion at $E_{\mathrm{B}} \sim 50 \mathrm{meV}$ (cyan dashed curves), which correspond to the $\alpha^{\prime}$ and $\beta$ bands, respectively, in Fig. 1(c) [note that the intensity of the inner $d_{x z / y z}$ band ( $\alpha$ band) is suppressed due to the matrix-element effect]. These bands intersect with each other to open a hybridization gap of $\sim 25 \mathrm{meV}$ caused by the spin-orbit coupling (SOC) [36] (the band dispersion after the hybridization is shown by red curves and the SOC-induced gap is highlighted by a red arrow). Intriguingly, the top of the $d_{x y}$ band gradually shifts upward with increasing $x$ and nearly touches $E_{\mathrm{F}}$ at $x=0.4$ 

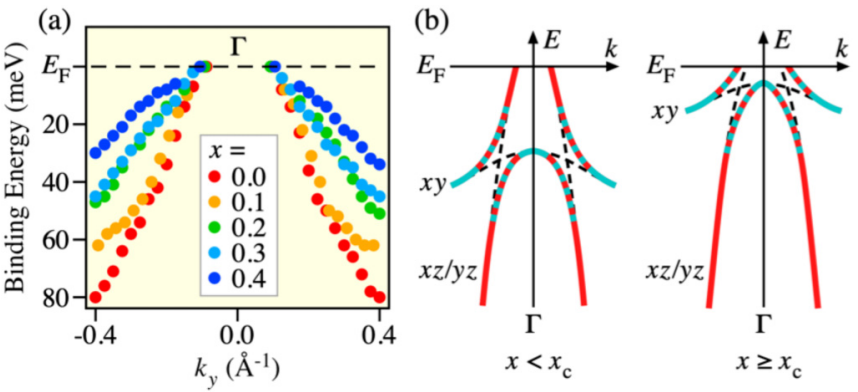

FIG. 3. (a) Comparison of the experimental near- $E_{\mathrm{F}}$ band dispersions extracted by tracing the peak position of ARPES spectra. (b) Schematics of the band structure around the $\Gamma$ point for $x<x_{\mathrm{c}}$ (left) and $x \geqslant x_{\mathrm{c}}$ (right).

(see also Fig. S2 in the Supplemental Material [35]). Due to such close proximity of the $d_{x y}$ band to $E_{\mathrm{F}}$, the electronic states at $E_{\mathrm{F}}$ are strongly modified for $x \geqslant 0.2$. Specifically, the hybridization of the $d_{x z / y z}$ band with the relatively flat $d_{x y}$ band causes flattening of the band dispersion near $E_{\mathrm{F}}$ [Figs. 2(h)-2(j)], resulting in a decrease of Fermi velocity $v_{\mathrm{F}}$ for $x \geqslant 0.2$, as corroborated by a direct comparison of the near- $E_{\mathrm{F}}$ band dispersions in Fig. 3(a). On the other hand, the electronic states at $E_{\mathrm{F}}$ are intact for $x \leqslant 0.1$ because the $d_{x y}$ band is well below $E_{\mathrm{F}}$ [Figs. 2(f) and 2(g)]. The quantitative analysis of experimental band dispersion in Fig. 3(a) shows that the $v_{\mathrm{F}}$ value for $x \geqslant 0.2$ is less than half that for $x \leqslant 0.1$, leading to an increase of the density of states (DOS) at $E_{\mathrm{F}}$.

Another important outcome of the upward $d_{x y}$-band shift is the participation of the $d_{x y}$ orbital with the holelike FS at $x \geqslant 0.2$. Although the top of the bare $d_{x y}$ band before the hybridization does not reach $E_{\mathrm{F}}$, this is enabled by the band hybridization. As schematically shown in Fig. 3(b), the band hybridization is expected to mix the $d_{x z / y z}$ and $d_{x y}$ orbitals in the $(E, \mathbf{k})$ region where the energy bands show a hybridization-induced shift. Thus, the observed clear change in the band dispersion at $E_{\mathrm{F}}$ (i.e., change in the $v_{\mathrm{F}}$ value) for $x \geqslant 0.2$ strongly suggests the finite $d_{x y}$-orbital component at $E_{\mathrm{F}}$ (right panel). On the other hand, a negligible contribution from the $d_{x y}$ orbital is expected at $E_{\mathrm{F}}$ for $x \leqslant 0.1$ because the influence of band hybridization does not reach $E_{\mathrm{F}}$ (left panel) [see also Figs. 2(f) and 2(g)]. Such consideration led us to conclude that the holelike FS consists of mixed $d_{x z / y z}+d_{x y}$ orbitals for $x \geqslant 0.2$, whereas it consists purely of the $d_{x z / y z}$ orbital for $x \leqslant 0.1$. We note that the upward energy shift of the $d_{x y}$ band could be satisfactorily explained in terms of an increase of chalcogen height from the Fe plane [37], which is naturally expected from the larger ionic radius of Te than that of Se [22].

Now we discuss implications of the present ARPES results in relation to the gigantic $T_{\mathrm{c}}$ enhancement. Our main findings are the suppression of nematicity near $x_{\mathrm{c}}$ and the energy shift of the $d_{x y}$ band. The former demonstrates that the high- $T_{\mathrm{c}}$ superconductivity anticorrelates with the nematicity in $\mathrm{FeSe}_{1-x} \mathrm{Te}_{x}$, as inferred from the anticorrelation between the superconductivity and the structural transition [32]. Such an anticorrelation implies that the two states are competing with each other or nematic/orbital fluctuations which potentially arise near the end point of the nematic phase may play (a)
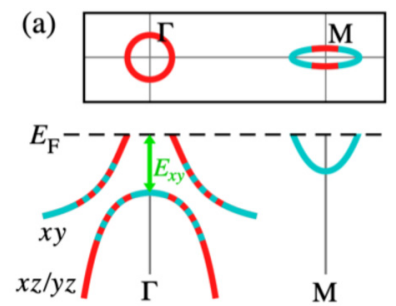

\begin{tabular}{l|l|l} 
& & \\
& & $\mathrm{M}$
\end{tabular}

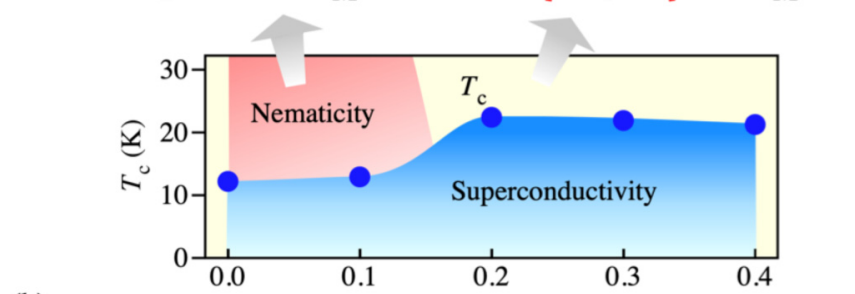

(b)

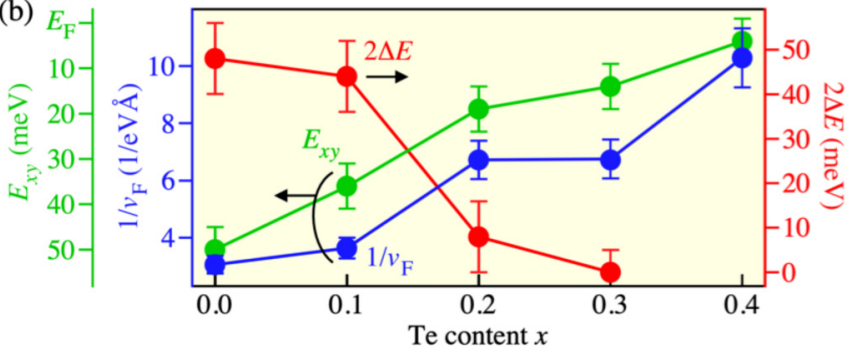

FIG. 4. (a) Schematic FSs and band dispersions at $T=30 \mathrm{~K}$ for $x<x_{\mathrm{c}}$ (left) and $x \geqslant x_{\mathrm{c}}$ (right), together with the phase diagram of $\mathrm{FeSe}_{1-x} \mathrm{Te}_{x} / \mathrm{CaF}_{2}$. (b) Comparison of the $x$ dependence of the nematic energy scale $2 \Delta E$ [red circles; same as the data in Fig. 2(r)], energy position of the $d_{x y}$-derived hole band at $\Gamma, E_{x y}$ (green circles), and an inverse of $v_{\mathrm{F}}$ for the hole pocket (blue circles).

a role in enhancing $T_{\mathrm{c}}$ [38-40]. Also, the latter finding, the $d_{x y}$-band shift, may be responsible for the $T_{\mathrm{c}}$ enhancement in two ways. First, as illustrated in the upper panel of Fig. 4(a) and also plotted by green circles in Fig. 4(b), the energy position of the $d_{x y}$-band top at the $\Gamma$ point with respect to $E_{\mathrm{F}}\left[E_{x y}\right.$, indicated by the green arrow in Fig. 4(a)] gradually decreases upon Te substitution, likely due to a gradual change in the anion height, as expected from the smooth evolution of lattice constants over a whole $x$ range [23] and, for $x \geqslant x_{\mathrm{c}}$, the $d_{x y}$ band is close enough to $E_{\mathrm{F}}$ to decrease $v_{\mathrm{F}}$ through the hybridization with the $d_{x z / y z}$ band, leading to an increase in the DOS at $E_{\mathrm{F}}$ around the $\Gamma$ point. A simple estimation by assuming a linear scaling between the DOS and $1 / v_{\mathrm{F}}$ indicates that the large enhancement of DOS coincides with the appearance of high- $T_{\mathrm{c}}$ superconductivity at $x=x_{\mathrm{c}}$ [see blue circles in Fig. 4(b)]. Therefore, the increase in the DOS (more than twice compared to that for $x<x_{\mathrm{c}}$ ) due to the decrease of $E_{x y}$ may contribute to the $T_{\mathrm{c}}$ enhancement at $x_{\mathrm{c}}$. We note that the increase of $1 / v_{\mathrm{F}}$ by a factor of $\sim 2$ is small compared to the large difference in the mass between the bare $d_{x z / y z}$ and $d_{x y}$ bands because $E_{\mathrm{F}}$ is located in the vicinity of their crossing point where the band velocity rather gradually changes. Second, small $E_{x y}$ also results in a transformation of the orbital character of the holelike FS from pure $d_{x z / y z}$ to mixed $d_{x z / y z}+d_{x y}$ near $x_{\mathrm{c}}$ [see the schematics in Fig. 4(a)]. The appearance of the $d_{x y}$ orbital on the holelike FS promotes the intra- $d_{x y}$-orbital scattering between the hole and electron 
pockets for $x \geqslant x_{\mathrm{c}}$ [indicated by a blue arrow in the upper right panel of Fig. 4(a)] and enhances spin fluctuations in the $d_{x y}$ channel. Such enhanced spin fluctuations in the $d_{x y}$ channel, either alone [37] or in cooperation with orbital fluctuations [38,39], have been proposed to increase $T_{\mathrm{c}}$. Therefore, our observations are compatible with the several routes to the $T_{\mathrm{c}}$ enhancement. Nevertheless, since $T_{\mathrm{c}}$ is usually determined by various parameters including effective interactions that are not solely controlled by the low-energy electronic structure, further experimental studies are desired to clarify the dominant source of high- $T_{\mathrm{c}}$ superconductivity, e.g., by determining the momentum/orbital dependence of the superconducting-gap size.

Next we discuss the possibility that the strength of nematicity is related to $E_{x y}$. The decrease of $E_{x y}$ causes not only the appearance of the $d_{x y}$-orbital state on the holelike FS, but also the reduction of the $d_{x z / y z}$-orbital state for $x \geqslant x_{\mathrm{c}}$, which in turn suppresses the spin fluctuation in the $d_{x z / y z}$ channel. According to the self-consistent vertex correlation theory, the reduction of spin fluctuation in the $d_{x z / y z}$ channel suppresses the nematicity because the orbital susceptibility is reduced through the orbital-spin interplay [39]. Although this theory is constructed to explain the phase diagram of FeSe under high pressure, the conceptually same situation is realized in $\mathrm{FeSe}_{1-x} \mathrm{Te}_{x} / \mathrm{CaF}_{2}$. Therefore, besides the large electronic and orbital reconstructions around the zone corner, the decrease of $E_{x y}$ around the zone center may play an additional role in suppressing the nematicity in $\mathrm{FeSe}_{1-x} \mathrm{Te}_{x} / \mathrm{CaF}_{2}$.
Finally, we comment on the role of SOC. As described above, when the SOC is absent, the $d_{x y}$ hole band seems to be located below $E_{\mathrm{F}}$ for all samples measured in this study. In other words, the appearance of the $d_{x y}$ state and the mass enhancement at $E_{\mathrm{F}}$ are realized not solely by the upward shift of $d_{x y}$ band but also by the assistance of SOC. Therefore, SOC may indirectly contribute to the increase in $T_{\mathrm{c}}$ and the suppression of nematicity at $x \geqslant x_{\mathrm{c}}$.

In conclusion, the present systematic high-resolution ARPES study has revealed a drastic energy shift of the $d_{x y}$ band which results in the participation of the $d_{x y}$ orbital in the formation of a holelike FS at $x \geqslant x_{\mathrm{c}}$. In addition, the suppression of nematicity has been found near $x_{\mathrm{c}}$, indicating the anticorrelation between the high- $T_{\mathrm{c}}$ superconductivity and the nematicity. These findings established distinct electronic structures with different orbital occupations between the pristine and high- $T_{\mathrm{c}} \mathrm{FeSe}_{1-x} \mathrm{Te}_{x} / \mathrm{CaF}_{2}$. The present results provide important insights into the interplay among high- $T_{\mathrm{c}}$ superconductivity, nematicity, and orbital degrees of freedom.

We thank Y. Kubota and K. Owada for their assistance with the ARPES measurements. We also thank UVSOR for access to beamlines BL7U and BL5U (Proposals No. 30-554, No. 19-568, and No. 19-857). This work was supported by JST-PRESTO (Grant No. JPMJPR18L7), JST-CREST (Grant No. JPMJCR18T1), and Grant-in-Aid for Scientific Research (JSPS KAKENHI Grants No. JP17H04847, No. JP17H01139, No. JP18H01160, No. JP19K14651, and No. JP20H01847).
[1] T.-M. Chuang, M. P. Allan, J. Lee, Y. Xie, N. Ni, S. L. Bud'ko, G. S. Boebinger, P. C. Canfield, and J. C. Davis, Science 327, 181 (2010).

[2] J.-H. Chu, J. Analytis, K. De Greve, P. McMahon, Z. Islam, Y. Yamamoto, and I. Fisher, Science 329, 824 (2010).

[3] M. A. Tanatar, E. C. Blomberg, A. Kreyssig, M. G. Kim, N. Ni, A. Thaler, S. L. Bud'ko, P. C. Canfield, A. I. Goldman, I. I. Mazin, and R. Prozorov, Phys. Rev. B 81, 184508 (2010).

[4] A. Dusza, A. Lucarelli, F. Pfuner, J. H. Chu, I. R. Fisher, and L. Degiorgi, Europhys. Lett. 93, 37002 (2011).

[5] M. Yi, D. Lu, J.-H. Chu, J. G. Analytis, A. P. Sorini, A. F. Kemper, B. Moritz, S.-K. Mo, R. G. Moore, M. Hashimoto, W.-S. Lee, Z. Hussain, T. P. Devereaux, I. R. Fisher, and Z.-X. Shen, Proc. Natl. Acad. Sci. U.S.A. 108, 6878 (2011).

[6] S. Kasahara, H. J. Shi, K. Hashimoto, S. Tonegawa, Y. Mizukami, T. Shibauchi, K. Sugimoto, T. Fukuda, T. Terashima, Andriy H. Nevidomskyy, and Y. Matsuda, Nature (London) 486, 382 (2012).

[7] J.-H. Chu, H.-H. Kuo, J. G. Analytis, and I. R. Fisher, Science 337, 710 (2012).

[8] R. M. Fernandes, A. V. Chubukov, and J. Schmalian, Nat. Phys. 10, 97 (2014).

[9] P. Dai, Rev. Mod. Phys. 87, 855 (2015).

[10] F.-C. Hsu, J.-Y. Luo, K.-W. Yeh, T.-K. Chen, T.-W. Huang, Y.-C. Lee, Y.-L. Huang, Y.-Y. Chu, D.-C. Yan, and M.-K. Wu, Proc. Natl. Acad. Sci. U.S.A. 105, 14262 (2008).

[11] T. M. McQueen, A. J. Williams, P. W. Stephens, J. Tao, Y. Zhu, V. Ksenofontov, F. Casper, C. Felser, and R. J. Cava, Phys. Rev. Lett. 103, 057002 (2009).
[12] K. Nakayama, Y. Miyata, G. N. Phan, T. Sato, Y. Tanabe, T. Urata, K. Tanigaki, and T. Takahashi, Phys. Rev. Lett. 113, 237001 (2014).

[13] T. Shimojima, Y. Suzuki, T. Sonobe, A. Nakamura, M. Sakano, J. Omachi, K. Yoshioka, M. Kuwata-Gonokami, K. Ono, H. Kumigashira, A. E. Böhmer, F. Hardy, T. Wolf, C. Meingast, H. v. Löhneysen, H. Ikeda, and K. Ishizaka, Phys. Rev. B 90, 121111(R) (2014).

[14] Y. Suzuki, T. Shimojima, T. Sonobe, A. Nakamura, M. Sakano, H. Tsuji, J. Omachi, K. Yoshioka, M. Kuwata-Gonokami, T. Watashige, R. Kobayashi, S. Kasahara, T. Shibauchi, Y. Matsuda, Y. Yamakawa, H. Kontani, and K. Ishizaka, Phys. Rev. B 92, 205117 (2015).

[15] P. Zhang, T. Qian, P. Richard, X. P. Wang, H. Miao, B. Q. Lv, B. B. Fu, T. Wolf, C. Meingast, X. X. Wu, Z. Q. Wang, J. P. Hu, and H. Ding, Phys. Rev. B 91, 214503 (2015).

[16] M. D. Watson, T. K. Kim, A. A. Haghighirad, N. R. Davies, A. McCollam, A. Narayanan, S. F. Blake, Y. L. Chen, S. Ghannadzadeh, A. J. Schofield, M. Hoesch, C. Meingast, T. Wolf, and A. I. Coldea, Phys. Rev. B 91, 155106 (2015).

[17] M. Yi, H. Pfau, Y. Zhang, Y. He, H. Wu, T. Chen, Z. R. Ye, M. Hashimoto, R. Yu, Q. Si, D.-H. Lee, P. Dai, Z.-X. Shen, D. H. Lu, and R. J. Birgeneau, Phys. Rev. X 9, 041049 (2019).

[18] P. O. Sprau, A. Kostin, A. Kreisel, A. E. Böhmer, V. Taufour, P. C. Canfield, S. Mukherjee, P. J. Hirschfeld, B. M. Andersen, and J. C. S. Davis, Science 357, 75 (2017).

[19] T. Hashimoto, Y. Ota, H. Q. Yamamoto, Y. Suzuki, T. Shimojima, S. Watanabe, C. Chen, S. Kasahara, Y. Matsuda, 
T. Shibauchi, K. Okazaki, and S. Shin, Nat. Commun. 9, 282 (2018).

[20] L. C. Rhodes, M. D. Watson, A. A. Haghighirad, D. V. Evtushinsky, M. Eschrig, and T. K. Kim, Phys. Rev. B 98, 180503(R) (2018).

[21] D. Liu, C. Li, J. Huang, B. Lei, L. Wang, X. Wu, B. Shen, Q. Gao, Y. Zhang, X. Liu, Y. Hu, Y. Xu, A. Liang, J. Liu, P. Ai, L. Zhao, S. He, L. Yu, G. Liu, Y. Mao et al., Phys. Rev. X 8, 031033 (2018).

[22] Y. Mizuguchi and Y. Takano, J. Phys. Soc. Jpn. 79, 102001 (2010).

[23] Y. Imai, Y. Sawada, F. Nabeshima, and A. Maeda, Proc. Natl. Acad. Sci. U.S.A. 112, 1937 (2015).

[24] J. Zhuang, W. K. Yeoh, X. Cui, X. Xu, Y. Du, Z. Shi, S. P. Ringer, X. Wang, and S. X. Dou, Sci. Rep. 4, 7273 (2014).

[25] S. Medvedev, T. M. McQueen, I. A. Troyan, T. Palasyuk, M. I. Eremets, R. J. Cava, S. Naghavi, F. Casper, V. Ksenofontov, G. Wortmann, and C. Felser, Nat. Mater. 8, 630 (2009).

[26] K. Matsuura, Y. Mizukami, Y. Arai, Y. Sugimura, N. Maejima, A. Machida, T. Watanuki, T. Fukuda, T. Yajima, Z. Hiroi, K. Y. Yip, Y. C. Chan, Q. Niu, S. Hosoi, K. Ishida, K. Mukasa, S. Kasahara, J.-G. Cheng, S. K. Goh, Y. Matsuda et al., Nat. Commun. 8, 1143 (2017).

[27] J. Guo, S. Jin, G. Wang, S. Wang, K. Zhu, T. Zhou, M. He, and X. Chen, Phys. Rev. B 82, 180520(R) (2010).

[28] Q.-Y. Wang, Z. Li, W.-H. Zhang, Z.-C. Zhang, J.-S. Zhang, W. Li, H. Ding, Y.-B. Ou, P. Deng, K. Chang, J. Wen, C.-L. Song, K. He, J.-F. Jia, S.-H. Ji, Y.-Y. Wang, L.-L. Wang,
X. Chen, X.-C. Ma, and Q.-K. Xue, Chin. Phys. Lett. 29, 037402 (2012).

[29] Y. Miyata, K. Nakayama, K. Sugawara, T. Sato, and T. Takahashi, Nat. Mater. 14, 775 (2015).

[30] G. N. Phan, K. Nakayama, K. Sugawara, T. Sato, T. Urata, Y. Tanabe, K. Tanigaki, F. Nabeshima, Y. Imai, A. Maeda, and T. Takahashi, Phys. Rev. B 95, 224507 (2017).

[31] M. Nakajima, K. Yanase, F. Nabeshima, Y. Imai, A. Maeda, and S. Tajima, Phys. Rev. B 95, 184502 (2017).

[32] Y. Imai, Y. Sawada, F. Nabeshima, D. Asami, M. Kawai, and A. Maeda, Sci. Rep. 7, 46653 (2017).

[33] F. Nabeshima, Y. Imai, M. Hanawa, I. Tsukada, and A. Maeda, Appl. Phys. Lett. 103, 172602 (2013).

[34] Y. Sawada, F. Nabeshima, Y. Imai, and A. Maeda, J. Phys. Soc. Jpn. 85, 073703 (2016).

[35] See Supplemental Material at http://link.aps.org/supplemental/ 10.1103/PhysRevResearch.3.L012007 for the evolution of the electronlike-FS shape and for the $d_{x y}$-band dispersion.

[36] S. V. Borisenko, D. V. Evtushinsky, Z.-H. Liu, I. Morozov, R. Kappenberger, S. Wurmehl, B. Büchner, A. N. Yaresko, T. K. Kim, M. Hoesch, T. Wolf, and N. D. Zhigadlo, Nat. Phys. 12, 311 (2016).

[37] K. Kuroki, H. Usui, S. Onari, R. Arita, and H. Aoki, Phys. Rev. B 79, 224511 (2009).

[38] Y. Yamakawa, S. Onari, and H. Kontani, Phys. Rev. X 6, 021032 (2016).

[39] Y. Yamakawa and H. Kontani, Phys. Rev. B 96, 144509 (2017).

[40] S. Lederer, Y. Schattner, E. Berg, and S. A. Kivelson, Phys. Rev. Lett. 114, 097001 (2015). 